\title{
Spectral Efficiency of Massive MIMO Communication Systems with Zero Forcing and Maximum Ratio Beamforming
}

\author{
Asif Ali ${ }^{1}$, Imran Ali Qureshi ${ }^{2}$, Abdul Latif Memon ${ }^{3}$, Sajjad Ali Memon ${ }^{4}$, Erum Saba ${ }^{5}$ \\ 1,2,3,4 Department of Telecommunication Engineering, ${ }^{5}$ Information Technology Center \\ 1,2,3,4 Mehran University of Engineering and Technology, ${ }^{5}$ Sindh Agriculture University \\ 1,2,3,4 Jamshoro, Pakistan, ${ }^{5}$ Tandojam, Pakistan
}

\begin{abstract}
The massive multiple-input-multiple-output (MIMO) is a key enabling technology for the 5G cellular communication systems. In massive MIMO (M-MIMO) systems few hundred numbers of antennas are deployed at each base station (BS) to serve a relatively small number of single-antenna terminals with multiuser, providing higher data rate and lower latency. In this paper, an M-MIMO communication system with a large number of $\mathrm{BS}$ antennas with zero-forcing beamforming is proposed for the improved spectral efficiency performance of the system. The zero forcing beamforming technique is used to overcome the interference that limits the spectral efficiency of MMIMO communication systems. The simulation results authenticate the improvement in the spectral efficiency of $M$ MIMO system. The spectral efficiency value using zero-forcing beamforming is near to the spectral efficiency value with the nointerference scenario.
\end{abstract}

Keywords-Massive MIMO; Base station; channel capacity; Spectral efficiency; latency; cellular communication; beamforming techniques; throughput; mobile communication

\section{INTRODUCTION}

In a few years, several studies have been focused on massive MIMO systems which play an important role in emerging trends in communication networks. The massive MIMO transceiver contains the various number of smart antenna arrays, which enable to get higher spectral efficiency and vigorously efficient to be achieved [1][2]. In various antennas were attached to an array form to organize the base station and mobile stations of the wireless communication link, in order to manage the signal in appropriate direction to improve the system performance [3]. The multi-beam forming needs to join the multiple signals received or transmitted by an array of antennas. Designing and implementation challenges are associated with its configuration and applications. The massive MIMO antennas are integrated with beamforming array antenna technologies for next-generation cellular communication and deployed in 2020 as recommended to [4]. Various numbers of communication model and antennas are unable to increase the capacity of wireless communication systems to mitigate the multipath fading and channel interference [5]. The beamforming technique is improving signal radiation accordingly to the environment. In communication networks, transmitter and receiver used the beamforming signal transmission from base station to mobile station with multiple antennas [6]. The key theme of enabling the beamforming in the communication networks, to enhance the power capacity and minimize the interference [7]. To transmit the same signal with various values of phase and amplitude, it can pass from the different MIMO channel was constructively added the desired signal and destructively on the other users. In MIMO array gain would contest the increased path losses but necessary to provide the suitable link budget [8]. The millimeter wave communication creates the massive MIMO (multi-input multi-output) system more smarts for the size of the antenna is small but makes a possible a huge number of array antennas in a small region at the BS and massive MIMO can conflict the high path loss and fading of mm-wave channels [9]. The massive MIMO principle is previously working in the $\mathrm{Wi}-\mathrm{Fi}$ and $4 \mathrm{G}$ standards, it will play a major role when the $5 \mathrm{G}$ networks are coming. Certainly, the massive MIMO is extensively estimated key enable technology and fundamental component of 5G [10][11].

\section{RELATED WORK}

In the related work of massive MIMO mostly consider the omnidirectional antennas for the base station. It is eminent to know that base station directional antennas are used along with sectorization antennas [12] to increase SINR of the cellular network. In massive MIMO multiples antennas are used, it poses the major challenges in it [13], a number of antennas are to increase the energy consumption and cost as well. In millimeter wave (mm-Wave) bands have high path loss at their operating frequencies which are essential for the high gain and sufficient to signal-to-noise ratio (SNR) at a very low distance [14]. In the low range wavelengths, of the mm-wave frequencies where the large antennas array is packed with small form factor [8], the higher gain array can be a conflict to increase the path loss and crucial to provide the suitable link budget [15]. Therefore, massive MIMO is a preferred technology to overcome these problems according to their desirable achievements.

\section{SYSTEM MODEL}

We have considered the uplink and downlink of one cell multi-user massive MIMO system consists of $\mathrm{K}$ number of single antenna users and BS prepared with $\mathrm{M}$ number of antennas. In our consideration, $\mathrm{k}$ is the all active users share the resources at the same time with perfect channel interference with base station end user [16]. Considering the 
multi-carrier frequency of flat channel and with symbol rates of sampling, the Mx1 signals received at the BS from the $\mathrm{K}$ users. In general, the propagation channels are modeled on large scale fading and small-scale fading, the zero mean and unit variances are estimated in this work. The postulation of the symbol rate of sampling is the matched at the receiver end; it must be executed in the analog sphere. The better presentation might be achieved by oversampling the ADC especially those of one-bit resolution [17]. The fading block representation through coherence bandwidth $W c$ and coherence time $T c$. In this model both channels are constant for an interval of length $T=T c W c$, those symbols were independently changed from various intervals. Note that $\mathrm{T}$ is a fixed for a minimum duration of all the users.

\section{Transmitter

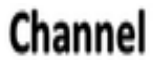

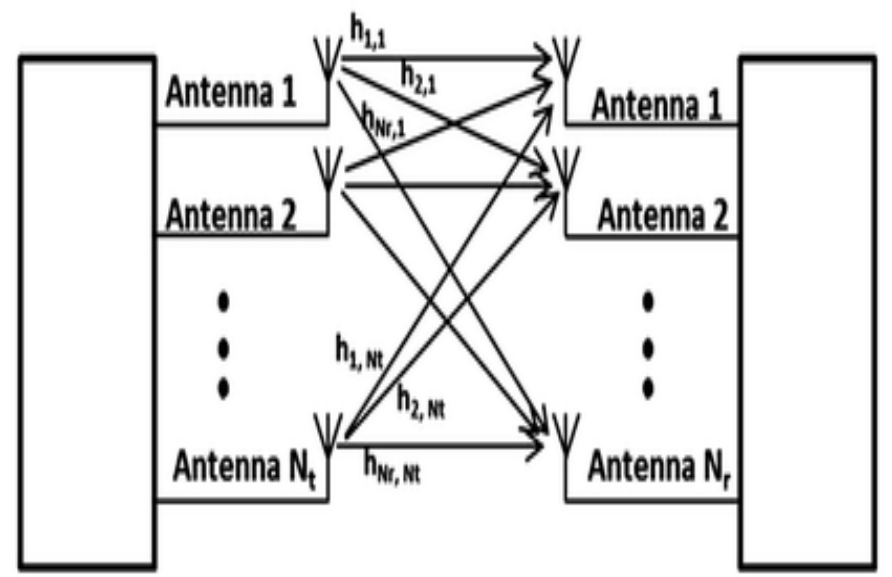

Fig. 1. Transmitter and Receiver System Model.

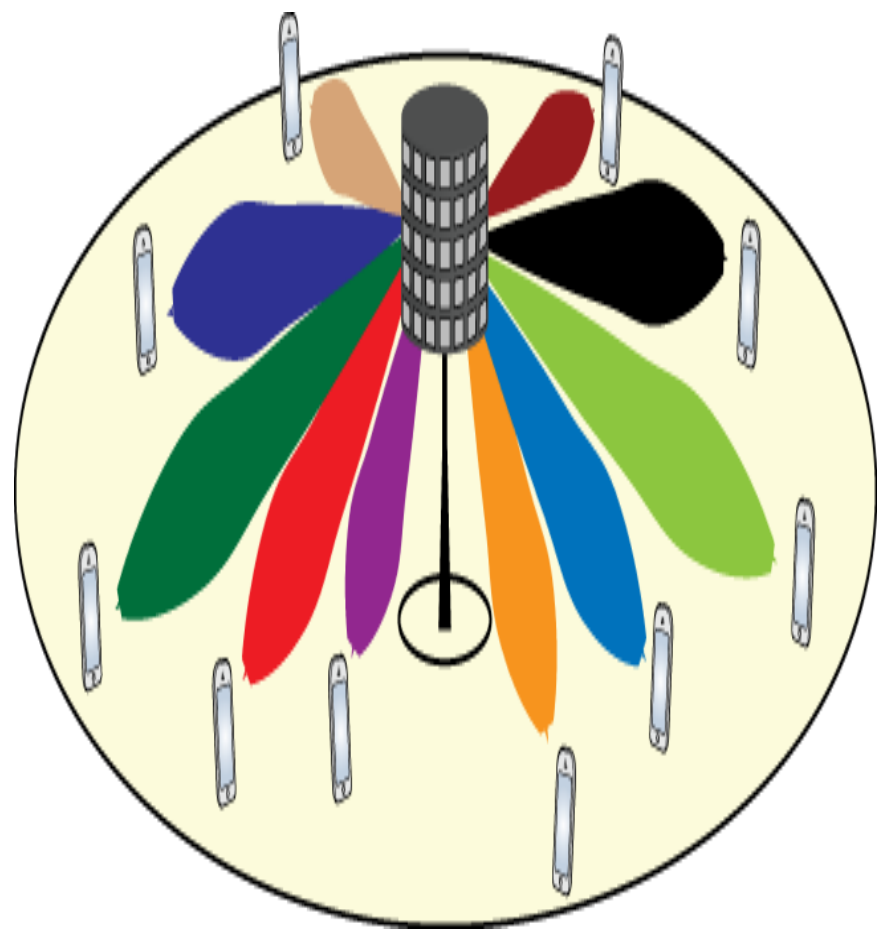

Fig. 2. The Uplink and Downlink Transmission in a Massive MIMO System.

\section{MASSIVE MIMO SYSTEM}

Massive MIMO is the primary component of the fast $5 \mathrm{G}$ networks in the future. Because the MIMO wireless networks allow the transmitting and receiving a large number of the data signal at the same time with the same radio channels. To fulfill the need of high data rates and good quality of service constraint, massive multiple-input-multiple-output (MIMO) systems will be preferred as they contain a la number of antennas at the access point, which is a suitable technology for $5 \mathrm{G}$ communication [18]. The massive MIMO technology is familiarized with the last two decades and useful to many wireless standards due to drastically improve the capability and reliability of wireless systems. The number of antennas is increased to massive MIMO to enhance the performance likewise: spectrum efficiency, energy efficiency and network coverage [19]. The massive MIMO systems are widely adopted in cellular communication as a vital component in the future. The array elements of massive MIMO systems are formulated to the shape of RF energy and reusing the spectrum between separated users. In this phenomenon, the energy falls into places outside the intended users, causing the unwanted interference with cell boundary and wasting the spectral power at the transmitter end. The MIMO technology utilizes entire bandwidth in multi-antenna BS spatially multiplex a large number of user workstation is compatible to communicate. The underlying principle of the Massive MIMO theory with its communication protocol is enlightened from a chronological point of view on [20] presents a fundamental theoretic performance analysis. The multi-cell simulate the ones were presented to showcase the massive MIMO ability may offer 100-fold improvements in spectral efficiency over existing tools, lacking for highly developed signal processing.

The massive MIMO system consists of base stations with $\mathrm{M}$ antennas that provides $\mathrm{K}$ single antenna terminal as shown in Fig.1 for graphic footprints of massive MIMO. The base station multiplexes the received the data stream per user for uplink and downlink. The base station uses the antennas to direct the signal in the direction of the preferred receiver in the downlink and to divide the multiple signals for the uplink transmission as given in Fig. 2. To mitigate interference and enhance the SNR just enabling extra antennas rather than sending multiple data streams [21]. The main advantage of the MIMO system over the existing network is that it can multiply the capability of wireless connectivity without need the additional spectrums. It is a considerable point that competence enhancement and it could potentially give up as a 100 -fold in the future.

\section{PROBLEMS AND ISSUES}

In massive MIMO systems, the major problem is interference while the antennas originate the same signal. To overcome this problem, 3D beamforming massive MIMO is a preferable solution over 2x2 Massive MIMO systems. In a massive MIMO system, pilot contamination is independent of the pilot sequence so it limits the reuse for uplink. The effect of reuse produce the conflict within different cells in the antenna array of the BS has correlated with the desired received pilot signal. The array antenna at the BS obtains a channel approximate that is corrupted by a combination of 
signals from other terminals using the same pilot sequence and it will create the interference. The hybrid beamforming is well-matched with mm-wave bands for massive MIMO systems. In comparison the lower frequency band MIMO systems which reduce the complexity and performance gap between beamforming techniques. The mm-wave and massive MIMO applications are limited due to LOS/near LOS properly. To mitigate the limitation of interference through superposition and beamforming techniques. The studies have focused on multiuser transmission schemes for massive MIMO systems. It is necessary to find the mm-wave channel propagation characteristics due to high path loss. The beamforming techniques and mm-wave system provide a solution to improve system performance. The source emits a signal and received at BS with estimated beamforming value for localization. The source is founded through triangulation and the dense multipath environment in the urban region. The localization based beamforming techniques may further be developed to get more benefits from it.

\section{ANTENNA PLACEMENT}

One of the major problems with a MIMO system is to replace the antennas. For many systems using actually undersized units, the antenna deployment offers some problem. To accurately run the MIMO system, the contact between antennas should be smaller. According to the thumb rule, $\lambda / 2$ spacing (where $\lambda$ is the signal wavelength) is measured essential to offer nearly no connection among the antennas. There are different methods to get taken. The optimal methods are commonly used in conservative small scale MIMO systems because the incredible number of antenna subsets is used in massive MIMO system which is necessary for antenna placement [22].

\section{A. Use High Frequencies}

To accommodate a large number of antennas are necessary for large MIMO systems, using the basic data onto frequency and wavelength as, high frequencies shorter the signal wavelength, therefore permit the dimension related antenna will be adjusted within the substantial gap. Many systems are taking into consideration the use of $10 \mathrm{GHz}$ frequency, to the extent that $60 \mathrm{GHz}$ and more [23].

\section{B. Use Volumetric Instead of Linear Spacing}

It is feasible to use the three dimensions inside a range of three dimension spacing as a linear fashion within two dimensions. Although there are many things that include mobile phones, are often slim and for that reason, this move toward cannot be appropriate, in some cases, a cube may be capable to adjust the maximum antennas with three dimensional spaced. The signal model and regard the effect of cluster size or spacing, the massive MIMO system contains the transmitter and receiver antennas [24].

\section{Use of Spatial Modulation}

The number of RF chains is necessary for a massive MIMO system can be condensed without understanding the spectral efficiency by spatial modulation. Spatial modulation is used to transmit a single chain for multiple antennas. It transmits the data and selects a single antenna from an array antenna for transmission at the same time. It has adopted an easy but effective coding mechanism, in which the bits of the transfer information and the local position of the transmitter antenna in the general antenna array. If the spreader signal reaches your destination at the receiving antenna is very low and the spacing antenna elements are inadequate the Massive MIMO capacity could be ruined. Therefore vigilant deliberation is needed for the antenna design and deployments [25].

\section{SIMULATION RESULTS}

In this section, the simulation results of the massive MIMO system for a cellular communication system with simulation parameters mentioned in Table I. To examine the numerical results where the number of users $k=10$ simultaneously provide the BS by M antennas. For the ease of all user is unspecified to have typical SNR of $5 \mathrm{~dB}$, there is an ideal channel state interference accessible everywhere. Fig. 3 illustrate that typical spectral efficiency like a purpose of $\mathrm{M}$ antennas, attain as a result of sum capability of non-linear processing and basic processing schemes known as the zeroforcing $(\mathrm{ZF})$, which crack to repress all obstruction. The uplink and downlink transmissions show in the form of results.

This simulation presents that the non-linear processing largely outperforms linear $\mathrm{ZF}$ when $M \approx K$. The working point $M=K$ creates exacting logic from a multiplexing perception for the multiplexing gain $\min (M ; K)$ does not develop, let $M$ raise for a fixed $K$. Fig. 3 demonstrate that there is some other cause to regard as $M>K$; the capability boosts and the performance will be linear $\mathrm{ZF}$ processing advance the capacity. Previously at $M=20$, there is only a little difference between linear $\mathrm{ZF}$ and optimum non-linear processing. Actuality, these two methods also approached the upper curve in Fig. 3, which characterizes the upper bounce which indicates where the hindrance among the users is ignoring. It illustrates the fundamentally that only $\mathrm{K}$ users can be served as every one of them was single handed in the cell.

\section{TABLE I. SiMULATION PARAMETERS}

\begin{tabular}{|l|l|}
\hline Simulation Parameters & Values \\
\hline Number of antennas(M) & $10-100$ \\
\hline Number of users(K) & 10 \\
\hline SNR & $5 \mathrm{~dB}$ \\
\hline Channel variance & Ones $(1, \mathrm{~K})$ \\
\hline Coherence time & 400 symbol \\
\hline
\end{tabular}

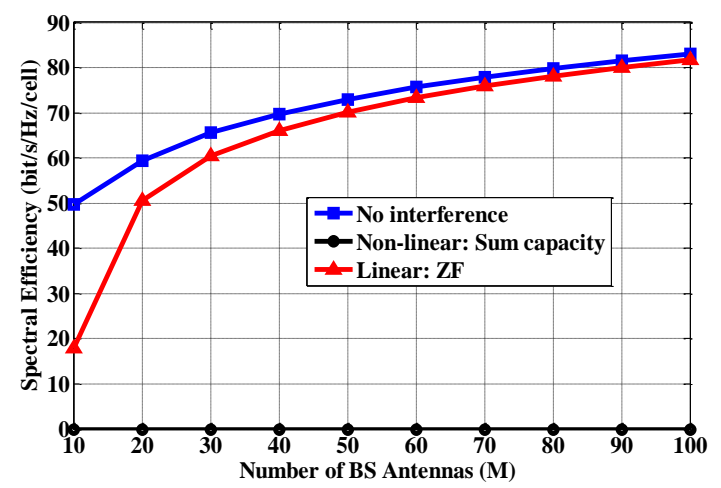

Fig. 3. The Average Spectral Efficiency of Massive MIMO for Both Uplink and Downlink. 


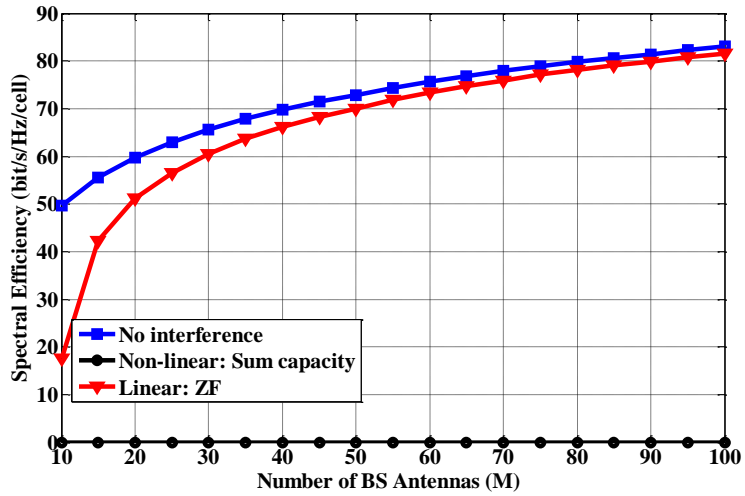

Fig. 4. An Improved Version of Spectral Efficiency for Uplink and Downlink Transmission.

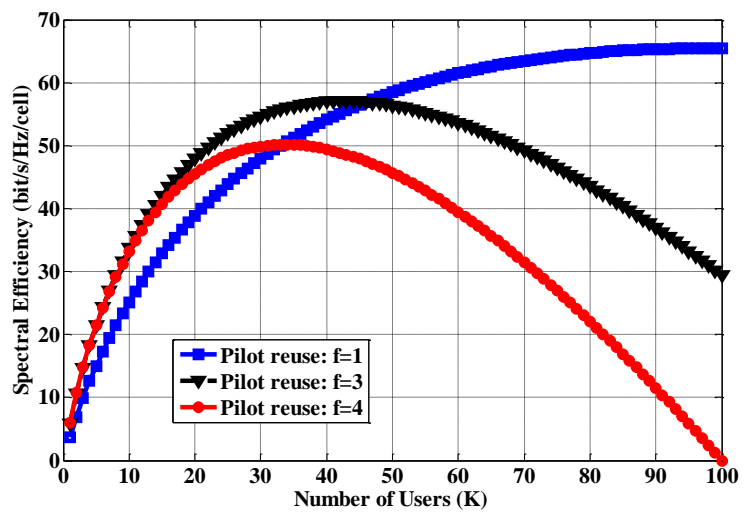

Maximum Ratio Detection.

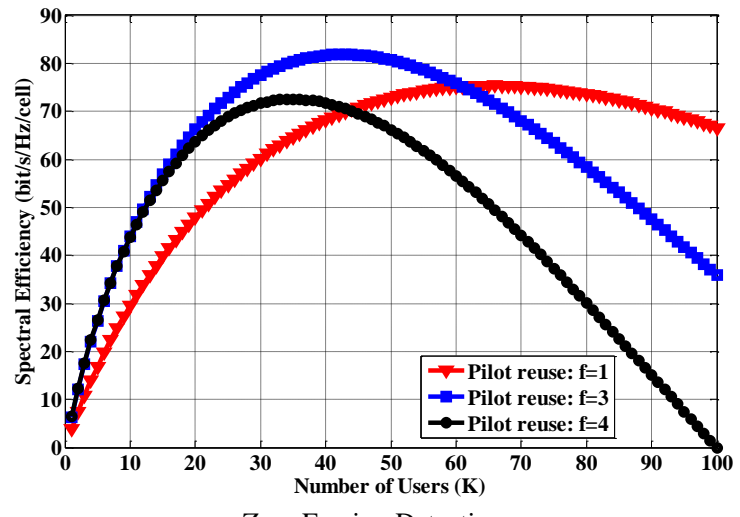

Zero Forcing Detection.

Fig. 5. Average Spectral Efficiency with different Pilot Reuse Factor at Two Different SNR Levels, OdB, and 20dB.

In this scenario, the $f=1$ shows the universal pilot reuse and $\mathrm{f}$ is greater than one known as the non-universal pilot reuse. In every tier has six cells of the hexagonal cell topology. The minimum reuse pilot feature improves the symmetric pilot reuse prototype are $\mathrm{f}=1, \mathrm{f}=3$, and $\mathrm{f}=4$. These reuse patterns are shown in Fig. 4, where different color shows different reuse pilot sequences. The same color of cells and same division of pilots, reason the pilot contagion to everywhere, while the other hand the different colors have no pilot contamination. We have noted the pilot reuse factor of $\mathrm{f}=4$, can be divided into four cells with different disjoint groups, or divide into subcells. Later on, this is recognized as the fractional pilot reuse and it has less common pilot reuse at the corners of the cell centers [26], due to its users at the cell borders are most susceptible to pilot contamination. In this scenario, massive MIMO contain $\mathrm{M}=200$ base station antennas and coherence time of $t c=400$ symbols. The users are unspecified to be regularly circulated in the cell and the channel is a representation as uncorrelated Rayleigh fading with non-line of sight attenuation with path loss exponent 3.7 [27].

The spectral efficiency of different users of both ZF and MR detection is shown in Fig. 5. In this inspection, the two SNR levels, $0 \mathrm{~dB}$, and $20 \mathrm{~dB}$ give the same performance. It noted that massive MIMO works similarly at both SNR levels whether its high or low, therefore array gain make it the interference limited and rather than noise limited. As the value of $\mathrm{f}$ improved properly it will operate top on the curve and massive MIMO can give the high spectral efficiency over a large number of users. When the number of users is raised like $\mathrm{K}>10$ as suggested in Fig. 6, it removes the complications from the massive MIMO networks because the active user serve the simultaneously in every coherence time interval of data, which number is typically more than hundreds and it shared between all the users. If the number of users increased beyond 200 then the spectral efficiency also decreases after a certain point, which is demonstrated in Fig. 7 that is an improved version of spectral efficiency.

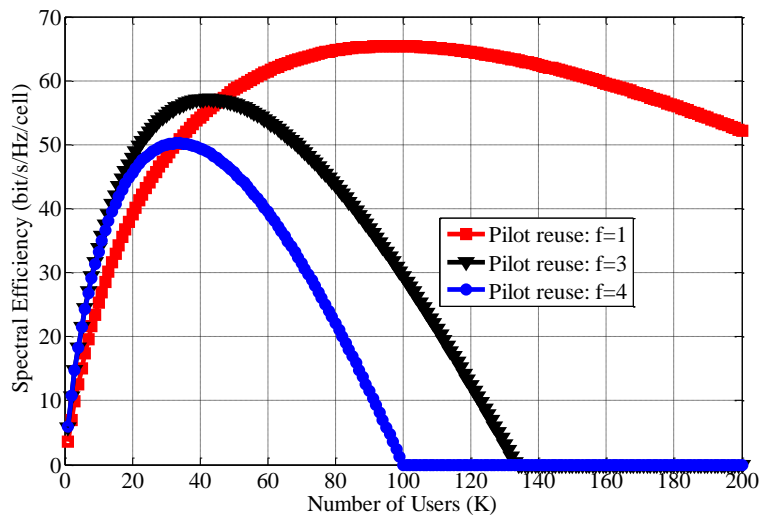

Maximum Ratio Detection.

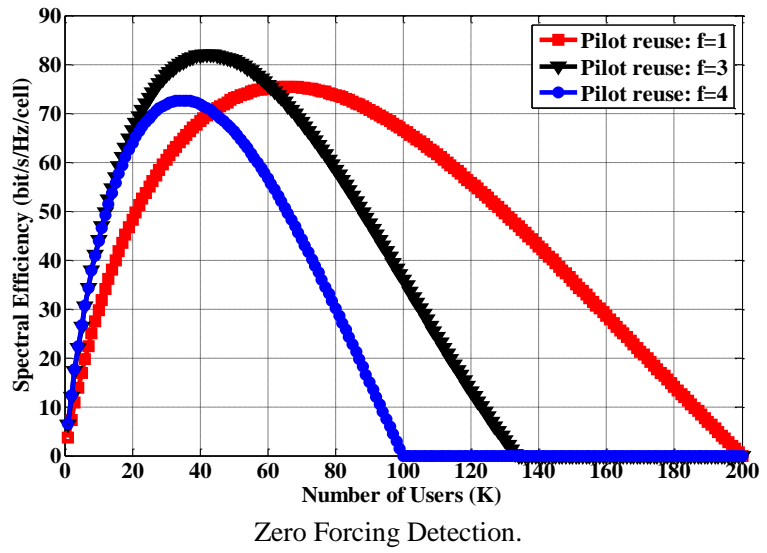

Fig. 6. Improved Spectral Efficiency at two Different Levels of SNR, OdB and $20 \mathrm{~dB}$ at $\mathrm{K}=200$ users. 


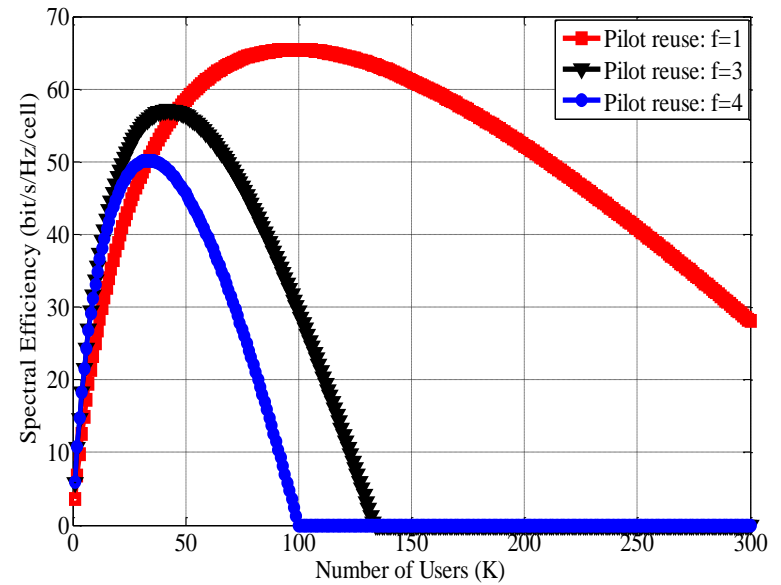

(a)

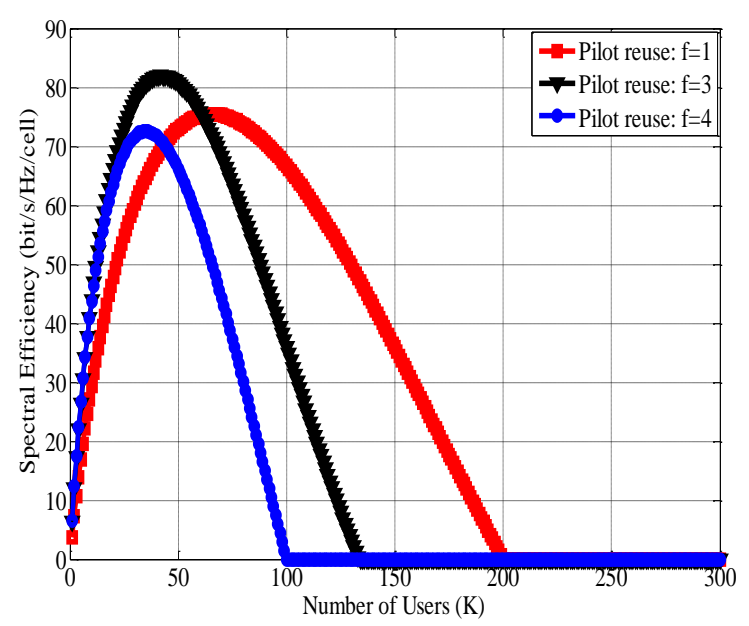

(b)

Fig. 7. Improved Spectral Efficiency and Pilot Reuse Factor at different Levels of SNR, $0 \mathrm{~dB}$ and $20 \mathrm{~dB}$ at $\mathrm{K}=300$ users.

The M-MIMO achieved a high sum of spectral efficiency which distributes among all users. The difference between MR and ZF in terms of gain is relatively small, the ZF provides the gain performance ratio between the $4 \%$ and $50 \%$ but depends on the user as well. In the single cell simulation where the zero-forcing offered more than twofold the spectral efficiency as compared to the MR. The cause for the unassuming performance space is suffered from ZF from the obstruction in the multicell given that the pilot contamination and countless inter-cell interferes very much possible to cancel all interferences. The pilot reuse factors are a very important parameter to design for the M-MIMO system and its good selection which depends on the user capability and propagation environment in which more number of antennas are used at the base station. The achieved spectral efficiency is 80 (bit/s/Hz/cell) and $82(\mathrm{bit} / \mathrm{s} / \mathrm{Hz} / \mathrm{cell})$ with 100 users using non-linear-sum capacity and no interference technique respectively, while maintaining same bandwidth of base stations as in current system. The pilot reuse is a limitation of spectral efficiency when the value of pilot reuse $(f=1)$ the efficiency is sustainable to provide better communication otherwise the efficiency decreases.

\section{CONCLUSION}

The massive MIMO technique introduces more efficiency in the present scenario of wireless communication systems. The single antenna was converted into arrays of antennas for advanced beamforming techniques; the major advantages are that it can be improving the SNR of the overall system along with data transmission within the cell during the time of interval. In the massive MIMO, it is necessary for linear detection to perform the zero-forcing to allow the reliable detection to accumulate high-quality performance through enabling an increase in the dimension of arrays which mitigate the processing problem and considered as a tolerable area. Massive MIMO sustainably increased the spectral efficiency and enhance capacity and coverage. A base station uses a large number of antennas in a massive MIMO system, therefore it achieves high throughput and more capacity. The small cell system uses the low power mini BS to avoid interference easily and enhance efficiency. Nowadays, it is used in smart cities and densely populated areas and not only suitable for improving the spectral efficiency but also higher throughput can be achieved in 5G technology.

\section{REFERENCES}

[1] H. Q. Ngo, S. Member, E. G. Larsson, S. Member, and T. L. Marzetta, "Energy and Spectral Efficiency of Very Large Multiuser MIMO Systems," vol. 61, no. 4, pp. 1436-1449, 2013.

[2] Y. Huang, S. Member, S. He, J. Wang, and S. Member, "Spectral and Energy Efficiency Tradeoff for Massive MIMO," IEEE Trans. Veh. Technol., vol. 67, no. 8, pp. 6991-7002, 2018.

[3] R. Choudhury, "A Network Overview of Massive MIMO for $5 \mathrm{G}$ Wireless Cellular: System Model and Potentials," Int. J. Eng. Res. Gen. Sci., vol. 2, no. 4, pp. 338-347, 2014.

[4] J. Huang, C. Wang, R. Feng, and J. Sun, "Multi-Frequency mmWave Massive MIMO Channel Measurements and Characterization for 5G Wireless Communication Systems," vol. 35, no. 7, pp. 1591-1605, 2017.

[5] L. I. Wang, J. Li, S. Member, J. Zhang, and S. Member, "Uplink Sum Rate Analysis of Massive Distributed MIMO Systems Over Composite Fading Channels," IEEE Access, vol. 6, pp. 25970-25978, 2018.

[6] E. Ali, M. Ismail, R. Nordin, and N. F. Abdulah, "Beamforming techniques for massive MIMO systems in $5 \mathrm{G}$ : overview, classification, and trends for future research," Front. Inf. Technol. Electron. Eng., vol. 18, no. 6, pp. 753-772, 2017.

[7] E. Björnson, J. Hoydis, and M. Kountouris, "Massive MIMO Systems With Non-Ideal Hardware : Energy Efficiency,Estimation, and Capacity Limits," vol. 60, no. 11, pp. 7112-7139, 2014.

[8] J. Jing, C. Xiaoxue, and X. Yongbin, "Energy-efficiency based downlink multi-user hybrid beamforming for millimeter wave massive MIMO system," J. China Univ. Posts Telecommun., vol. 23, no. 4, pp. 53-62, 2016.

[9] O. El Ayach and R. W. Heath, "Multimode Precoding in MillimeterWave MIMO Transmitters with Multiple Antenna Sub-Arrays," pp. 3476-3480, 2013.

[10] Sajjad Ali, Z. Chen and F. Yin "Eradication of pilot contamination and zero forcing precoding in the multi-cell TDD massive MIMO systems" IET Communications vol. 11 no.13, pp. 2027-2034, 2017.

[11] Sajjad Ali, Zhe Chen, and Fuliang Yin "Pilot decontamination in TDD multi-cell massive MIMO systems with infinite number of BS antennas" Canadian Journal of Electrical and Computer Engineering (IEEE Canada), vol. 40, no. 3, pp. 171-180, Summer 2017.

[12] S. Shahsavari, P. Hassanzadeh, A. Ashikhmin, and E. Erkip, "Sectoring in multi-cell massive MIMO systems," Conf. Rec. 51st Asilomar Conf. Signals, Syst. Comput. ACSSC 2017, vol. 2017-October, pp. 10501055, 2018. 
[13] A. F. Molisch, V. V. Ratnam, S. Han, Z. Li, S. L. H. Nguyen, L. Li, and K. Haneda, "Hybrid Beamforming for Massive MIMO: A Survey," IEEE Commun. Mag., vol. 55, no. 9, pp. 134-141, 2017.

[14] E. G. Larsson, O. Edfors, F. Tufvesson, and T. L. Marzetta, "Massive MIMO for next generation wireless systems," IEEE Commun. Mag., vol. 52, no. 2, pp. 186-195, 2014.

[15] S. Rajoria, A. Trivedi, and W. W. Godfrey, "A comprehensive survey: Small cell meets massive MIMO,” Phys. Commun., vol. 26, pp. 40-49, 2018.

[16] H. Wang, D. Yang, X. Li, and P. Pan, "How Many Signals Can Be Sent in a Multi-Cell Massive MIMO System," IEEE Wireless. Commun. Lett., vol. 7, no. 3, pp. 368-371, 2018.

[17] H. Pirzadeh, S. Member, and A. L. Swindlehurst, "Spectral Efficiency of Mixed-ADC Massive MIMO," no. c, pp. 1-15, 2018.

[18] X. Wei et al., "Uplink Channel Estimation in Massive MIMO Systems Using Factor Analysis," vol. 7798, no. c, pp. 1-4, 2018.

[19] L. Lu, S. Member, G. Y. Li, and A. L. Swindlehurst, "An Overview of Massive MIMO : Benefits and Challenges," vol. 8, no. 5, pp. 742-758, 2014.

[20] T. Van Chien, S. Member, E. Björnson, S. Member, and E. G. Larsson, "Joint Pilot Design and Uplink Power Allocation in Multi-Cell Massive MIMO Systems," vol. 17, no. 3, pp. 2000-2015, 2018.
[21] E. Björnson, M. Kountouris, M. Bengtsson, and S. Member, "Receive Combining vs. Multi-Stream Multiplexing in Downlink Systems With Multi-Antenna Users," vol. 61, no. 13, pp. 3431-3446, 2013.

[22] C. Bounds, "Massive MIMO Antenna Selection: Switching," vol. 66, no. 5, pp. 1346-1360, 2018.

[23] L. Zhao, K. Li, K. Zheng, S. Member, and M. O. Ahmad, "An Analysis of the Tradeoff Between the Energy and Spectrum Efficiencies in an Uplink Massive MIMO-OFDM System,” vol. 62, no. 3, pp. 291-295, 2015.

[24] Y. Yang et al., "Fast Optimal Antenna Placement for Distributed MIMO Radar with Surveillance Performance," vol. 22, no. 11, pp. 1955-1959, 2015.

[25] A. E. Forooshani, A. A. Lotfineyestanak, S. Member, D. G. Michelson, and S. Member, "Optimization of Antenna Placement in Distributed MIMO Systems for Underground Mines," vol. 13, no. 9, pp. 4685-4692, 2014.

[26] Italo Atzeni, Jesus Arnau, and Merouane Debbah "Fractional Pilot Reuse in Massive MIMO Systems," IEEE ICC, pp. 1030-1035, 2015.

[27] L. Shen, Y. Yao, H. Wang, H. Wang, and S. Member, "ICA Based Semi-Blind Decoding Method for a Multicell Multiuser Massive MIMO Uplink System in Rician / Rayleigh Fading Channels," vol. 16, no. 11, pp. 7501-7511, 2017. 